\title{
Plateformes d'innovation comme dispositif d'orientation des trajectoires technologiques des filières agricoles. Cas de la filière banane plantain en Côte d'Ivoire
}

\section{Innovation Platforms as a Tool to Support Technological Change in Agri-}

\author{
Chains. The case Study of Plantain Value Chain in Côte d'Ivoire
}

\author{
Euphrasie Angbo-Kouakou ${ }^{1,2}$, Ludovic Temple ${ }^{1}$, Syndhia Mathé ${ }^{1,3}$, Alexandre Assemien ${ }^{2}$
}

${ }^{1}$ UMR Innovation, Centre de coopération internationale en recherche agronomique pour le développement, CIRAD, Montpellier, euphrasie.angbo@cirad.fr, ludovic.temple@cirad.fr, syndhia.mathe@cirad.fr

2 Institut National Polytechnique Félix Houphouët-Boigny (INP-HB), École Doctorale Polytechnique (EDP), UMRI 88, Laboratoire de Droit, d'Économie et de Gestion (LADEG), Yamoussoukro, Côte d'Ivoire, alexassem@yahoo.fr

${ }^{3}$ IITA, Yaoundé, Cameroon

RÉSUMÉ. Les politiques d'innovation dans les filières agricoles en Côte d'Ivoire depuis 2011 reposent sur la création d'un dispositif de transferts technologiques qualifié de "Plateformes d'Innovation", pour introduire des plants de variétés et d'hybrides améliorés. Cet article s'intéresse particulièrement aux conséquences des "Plateformes d'Innovation Banane Plantain" (PIP) dans la réorientation des choix technologiques locaux. II interroge leurs résultats sur l'amélioration de l'indépendance alimentaire. Nous utilisons le cadre conceptuel des Systèmes Sectoriels d'Innovation (SSI) pour caractériser le fonctionnement de ces PIP. Nous identifions quatre composantes qui structurent ces innovations socio-technologiques: la composante recherche, la composante intermédiation, la composante chaîne de valeur et la composante financement. Nos résultats montrent que ces PIP restructurent le SSI car elles déterminent de nouvelles orientations des politiques publiques (recherches et innovation) dans le choix des cultivars introduits, des pratiques culturales et des préférences alimentaires, en intégrant la diversité géographique des bénéficiaires. Ces réorientations impliquent la prise en compte des besoins des producteurs et des consommateurs en matière de choix du matériel végétal. Le futur de ces PIP s'en trouve alors interrogé.

ABSTRACT. Since 2011, innovation policies in the agri-chains of the Ivory Coast have been based on tools of technology transfer, known as "Innovation Platforms", for the introduction of plants of improved varieties or of plant hybrids. This article focuses primarily on the implications of "Plantain Innovation Platforms" (PIPS) in the reorientation of local technologies choices and ultimately in food independence through improved domestic food supplies. We use the conceptual framework of Sectoral System of Innovation (SSI) to characterize the functioning of PIPs. We identify four components which structure this socio-technological innovations: a research component, a dissemination component, a value chain component and a financing component. Our results shows that PIPs help to structure the SSI by influencing the public policy decision process (research and innovation) in the selection of the introduced cultivars, the cultural practices and also the food preferences, integrating geographic diversity of recipients of these innovations. These policy changes involve considering the needs of local producers and consumers concerning the choice of plant material and providing new technical choices. The future of these PIPs is thereby questioned.

MOTS-CLÉS. Trajectoires technologiques, Plateformes d'Innovation Plantain, Système Sectoriel d'Innovation, Nouvelles variétés d'hybrides, Côte d'Ivoire.

KEYWORDS. Technological change, Plantain Innovation Platforms, Sectoral System of the Innovation, New hybrid varieties, Ivory Coast.

\section{Introduction}

Le renouvellement des politiques publiques en Afrique de l'Ouest depuis quelques années, réhabilite la nécessité d'accroitre la sécurité alimentaire sans dépendre des marchés internationaux, donc à travers une augmentation de la production vivrière au regard des besoins du marché intérieur (HUGON 1994), (BRICAS 2013). Ces politiques sont soutenues par le Programme de Productivité Agricole de l'Afrique de l'Ouest (PPAAO, en anglais WAAPP), initié depuis 2011 par la Communauté Économique des États de l'Afrique de l'Ouest (CEDEAO) avec l'appui financier de la Banque Mondiale. Ces programmes concernent notamment la génération et / ou le transfert d'innovations technologiques (races pures d'animaux et variétés ou hybrides de végétaux : cultivars), ainsi que leur 
diffusion auprès des populations et publics-cibles dans les filières (TEMPLE, LANÇON, PALPACUER et PACHE 2011a) agricoles et alimentaires dans cette sous-région.

En Côte d'Ivoire, les actions sont engagées pour le compte de l'État par le Fonds Interprofessionnel pour la Recherche et le Conseil Agricole (FIRCA), et une agence d'exécution technique et fiduciaire (FIRCA_WAAPP_Côte d'Ivoire). Dans ce pays, le programme WAAPP se déroule sous la tutelle du Ministère en charge de l'Agriculture en collaboration avec une vingtaine d'institutions nationales et internationales (CGIAR 2013) notamment de recherche et des partenaires techniques et financiers. Un des objectifs de ce programme d'amélioration de la productivité agricole et alimentaire est d'établir les conditions pour le développement des systèmes d'innovation agricole (SIA) par la sélection et l'introduction de nouvelles variétés et hybrides améliorés dans les plantations de cultures vivrières.

Un élément central de fonctionnement de ces systèmes d'innovation institués par différents projets et programmes en Afrique sub-saharienne (ASS), porte sur la création de plateformes d'innovation et d'échanges multi acteurs (NEDERLOF.,et al.,2011), (ADEKUNLE et FATUNBI 2012), (KLERKX, VAN MIERLO et LEEUWIS 2012), (KILELU et al. 2013), (SCHUT et al., 2015). Ces plateformes ont pour missions d'organiser les articulations entre parties prenantes pour utiliser les résultats de la recherche et de l'innovation dans les domaines de l'agriculture et de l'alimentation. Elles ont ainsi pour objectifs de créer des cadres de transfert, de vulgarisation, de co-construction, de co-production des technologiques, et / ou de concertation entre acteurs, pour venir en support aux innovations en vue du développement d'une filière agroalimentaire.

Toutefois, le changement de trajectoires technologiques sous-entend la coévolution de trois dimensions de l'innovation notamment «le hardware, le software et l'orgware » (KLERKX et al., 2012). Les approches basées sur le diffutionisme ont cependant tendance à mettre l'accent sur le hardware et très peu sur le software et l'orgware. Pour preuve, des technologies existent depuis longtemps mais n'ont pas encore été diffusées. La stratégie actuelle est de travailler sur le software et l'orgware pour faire évoluer les trajectoires technologiques. Il s'agit donc d'examiner comment les plateformes d'innovation peuvent aider à cela. Il se pose également la question de comprendre en quoi les plateformes d'innovation contribuent à structurer des Systèmes Sectoriels d'Innovation (SSI) (MALERBA 2005) et réorientent des choix technologiques dans les filières agricoles et agroalimentaires en Côte d'Ivoire.

Il importe de noter que la dimension "hardware" de l'innovation ramène ici conjointement à une technologie spécialement impulsée par la recherche agronomique et aux dispositifs technologiques qui viennent en support à l'innovation tels que les plateformes d'innovation. La dimension "software" désigne les connaissances transmises par les services d'intermédiation (conseil) aux bénéficiaires à travers les séances de formation, d'apprentissage et de renforcement de capacités des bénéficiaires de ces innovations. La dimension "orgware" renverrait ainsi aux stratégies de coordination des acteurs de la filière et à la restructuration des composantes d'un SSI agricole (SSIA) à travers les échanges entre structures de recherche, services de vulgarisation ou d'appui conseil et bénéficiaires / acteurs cibles.

Nous proposons dans cette étude d'évaluer l'impact de ces dispositifs de transferts (plateformes) sur l'organisation et l'évolution d'un SSIA caractérisé par des institutions de recherche, des organismes financiers, des professionnels de la filière et des services d'intermédiation assurant la vulgarisation agricole; et leur incidence sur l'orientation des trajectoires technologiques de recherche et d'innovation. Il s'agit également d'analyser la capacité de ce dispositif à inclure différentes catégories d'acteurs d'une filière tels que les producteurs, les commerçants et les transformateurs, pour un développement adapté à la diversité géographique des régions de production agricole dans ce pays.

Le cadre méthodologique utilise l'approche SSI (MALERBA 2005), (TOUZARD et al., 2015) afin de représenter les relations entre nos quatre composantes de ce dispositif sectoriel de transfert technologique : la composante recherche, la composante intermédiation, la composante chaîne de 
valeur et la composante financement. L'analyse de cette innovation socio-technologique vérifie comment les dynamiques multi-acteurs structurent un SSIA et coordonnent les acteurs dans les cinq Plateformes d'Innovation Plantain (PIP) existantes dans une chaîne globale de valeur (GEREFFI et al., 2005) ou une filière agricole en Côte d'Ivoire.

L'étude de cas référencée s'appuie sur l'utilisation de données d'enquêtes qualitatives réalisées par le biais de focus groupe, d'entretiens semi-directifs et d'enquêtes auprès des différents acteurs identifiées dans chacune de ces composantes (Ministère de l'agriculture, MINADER; Agence d'intermédiation, ANADER (Agence Nationale de Developpmeent Rural); Agence d'exécution du programme WAAPP, FIRCA; Centre de coordination des programmes de recherche agronomique, CNRA ; etc.), ainsi qu'auprès des leaders et responsables en charge des PIP implantées dans quatre des principales régions productrices de la banane plantain (Abengourou, Adzopé, Agboville, et Soubré). Le matériel de collecte a été complété avec une dizaine d'entretiens réalisés auprès de chercheurs et d'experts du secteur vivrier basés en France et en Côte d'Ivoire entre 2015 et 2016.

L'hypothèse de recherche stipule que la création de plateformes collaboratives ou de transferts est un élément structurant d'existence de ce système d'innovation. Notre argumentaire s'appuie sur deux hypothèses secondaires de travail. Premièrement, nous supposons que les plateformes, en faisant interagir professionnels et institutions de recherche réadaptent les processus d'innovation et les apprentissages collectifs à la diversité des contextes. Deuxièmement, que ces plateformes réorientent les mécanismes de créations ou d'introductions variétales opérés par les structures nationales de recherche agronomique à travers un processus rétroactif sur le SSIA.

Cet article est organisé en quatre sections. Dans la première section, nous exposons les évolutions de l'introduction des innovations technologiques dans la filière banane plantain en Côte d'Ivoire. La deuxième section décrit le cadre d'analyse des SSI que nous mobilisons et les caractéristiques des quatres composantes identifiées, ainsi que la méthodologie retenue pour la collecte des données. Dans la troisième section, nous présentons nos résultats qui sont discutés dans la quatrième section. En conclusion, nous formulons des recommandations et les perspectives de recherches futures.

\section{Les innovations technologiques dans la filière banane plantain ivoirienne}

\section{1. Évolution des transferts de cultivars de bananiers plantains}

La revue de la littérature sur l'introduction des variétés et hybrides de bananiers plantains en Côte d'Ivoire et sur le processus de création des Plateformes d'Innovation de banane Plantain (PIP) met en exergue les éléments macroéconomiques, les aspects de politiques publiques, de programmes ou projets qui ont construit les transferts de cultivars depuis les années 80 (LASSOUDIERE 1973, 1989), (CHATAIGNER et TANO 1980), (OSSENI 1998), (KOFFI 2001, 2004), (CNRA 2008), (TRAORÉ, et al., 2009).

Cette littérature explique l'évolution de l'environnement socio-institutionnel des processus d'innovation dans le secteur vivrier (CHALEARD 1996), (PNIA 2010), (PNSAN 2011), (SNDVC 2014), (BLONDEL et al., 2014), (PERRIN 2015).

Dans le cadre présent, nous nous intéressons aux dernières introductions de trois (3) cultivars améliorés de plants de bananiers plantains : PITA 3 (2012), FHIA 21 (2012) et Big Ebanga (2014). Le traitement de ces informations secondaires permet d'identifier les composantes d'un SSI agricole (SSIA) sur la banane plantain, en construction pour la Côte d'Ivoire. 
L'environnement socio-institutionnel de cette filière est alors caractérisé ${ }^{1}$ pour échantillonner les acteurs majeurs ou parties prenantes qui ont été enquêtés.

\subsection{Historique de l'intervention du programme WAAPP_Banane Plantain}

En 2012, les producteurs de la filière banane plantain de Côte d'Ivoire ont été informés de l'existence de nouvaux cultivars améliorés de bananiers plantains à haut rendement et potentiellement tolérant à la Cercosporiose (une maladie foliaire) à savoir le PITA 3 et le FHIA 21. Ces deux hybrides ont fait par la suite l'objet d'un test expérimental dans dix groupements de producteurs situés dans sept zones que sont : Abengourou (2), Bouaflé (2), Issia (2), Adzopé, Agboville, Divo et Tiassalé, ce qui représenterait un total de 224 producteurs bénéficiaires dont 155 femmes, soit plus de $69 \%$ (WAAPP INFO5 2013). Ces distributions se sont poursuivies en 2013 et ont continué jusqu'en 2014. Environ 92680 plants de PITA 3 et FHIA 21 ont été gratuitement distribués à plus de 160 groupements de producteurs entre 2012 et 2014 par l'ANADER, qui assure l'appui conseil (vulgarisation) dans les localités de production agricole, l'un des partenaires priviligiés au developpement du monde rural.

Les parcelles des tests de démonstration étaient subdivisées en trois compartiments dans chaque groupement et couvraient en moyenne une superficie d'un quart (1/4) d'hectare (ha) devant accueillir les deux hybrides améliorés sujettes à la diffusion et une variété locale (Affoto / N'Dè Fôtô ou Agnrin / Agninnin) servant de témoin, tous sélectionnés par la recherche agronomique (CNRA) ${ }^{2}$. Cela en guise de comparaison des méthodes culturales. Sur la parcelle témoin, les producteurs avaient la latitude de procéder à la culture du bananier plantain selon les pratiques culturales traditionnelles; tandis que sur les parcelles supportant les cultivars d'hybrides améliorés, il s'agissait d'appliquer de nouvelles techniques culturales plus spécifiques en matière de conduite d'une exploitation agricole en monoculture, sous la supervision des agents de vulgarisation et de conseil de l'ANADER.

L'utilisation des plants de bananiers plantains sélectionnés par la recherche modifie radicalement les techniques culturales locales (TEMPLE et al., 2011b). Elle réduit la sensibilité aux maladies et donc accroît le rendement à l'hectare selon les chercheurs, ce qui a été confirmé par les producteurs enquêtés.

À la suite de cette expérimentation, cinq PIP ont été créées en 2013 dans cinq des principales régions de production de la banane plantain : Abengourou, Adzopé, Agboville, Issia et Soubré, à travers le financement du projet WAAPP sur la base d'un partenanriat pubic - privé (PPP) entre l'État ivoirien et la Banque Mondiale.

\footnotetext{
1 Les institutions nationales impliquées dans ce processus d'innovation sur la banane plantain sont notamment: le Ministère en Charge de l'Agriculture (MINADER) et celui de l'Économie et des Finances (MEF), le Fonds Interprofessionnel pour la Recherche et le Conseil Agricole (FIRCA), le Centre National de Recherche Agronomique (CNRA), I'Ivoirienne de Technologie Tropicale (I2T), le Centre National de Spécialisation en Banane Plantain (CNS BP), I'Institut National Polytechnique Félix Houphouët Boigny (INPHB), l'École Supérieur d'Agronomie (ESA_INPHB), l'École Nationale de la Statistique et Économie Appliquée (ENSEA), I'Agence Nationale de Développement Rural (ANADER), I'Institut Africain pour le Développement Économique et Social (INADES-Formation), les Plateformes d'Innovation de Banane Plantain (PIP), I'Association pour le Développement des Cultures Intensives (ADCVI), les Organisations Professionnelles Agricoles (OPA), I'ONG Rongead_International Trade \& Sustainable Development, I'ONG Chigata_ Femmes et développement), etc. Les partenariats extérieurs sont établis avec le CIRAD, le CGIAR, l'IITA et le CARBAP.
}

\footnotetext{
2 En 2007, les hybrides PITA 3, FHIA 21 et CRBP 100, ont fait l'objet d'une sélection participative des plants de bananiers plantains dans l'ouest de la Côte d'Ivoire. Ce test pilote a motivé, selon le CNRA, le choix des variétés PITA 3 et FHIA 21 dans la mise en œuvre du projet WAAPP. http://www.cnra.ci/downloads/Rap_programmes\%20de\%20recherche\%202007.pdf_p57.
} 


\subsection{Caractéristiques des plateformes : Objectifs, Compositions et Gouvernance théorique}

\subsubsection{Objectifs étatiques visés à la création des plateformes d'innovation}

La recherche agronomique dispose d'un certain nombre de technologies pour lesquelles l'efficacité a été prouvée en station expérimentale mais pas toujours en milieu réel. Ainsi, en mettant en place les plateformes d'innovation dans les filières agricoles et alimentaires (plantain, manioc, maïs et riz), les autorités ivoiriennes ambitionnent d'intégrer les besoins réels des acteurs économiques (producteurs, transformateurs, etc.) pour améliorer le transfert des résultats de la recherche.

Les objectifs initiaux de ces plateformes d'innovation sont principalement de faciliter l'accès aux intrants agricoles (hybrides), d'augmenter la production de ces cultures stratégiques (PNIA 2010), de soutenir l'écoulement de ces produits sur les marchés locaux et régionaux, et surtout de construire un cadre permanent de concertation multi parties prenantes dans les filières agroalimentaires ivoiriennes.

Les PIP constituent alors un nouveau dispositif technologique (hardware) mis en place dans le cadre de la réalisation des programmes d'amélioration de la productivité agricole de la banane plantain, qui expérimentalement structure le SSIA dans la filière en faisant évoluer les dimensions "orgware" et "software" de l'innovation. Elles viennent assurer dans ce processus la synergie entre acteurs de cette filière, tout en élargissant le spectre des acteurs à tous les différents maillons de la chaîne de valeur.

La conception des PIP ambitionne ainsi l'accroissement de l'offre domestique de vivriers locaux pour améliorer l'indépendance alimentaire en Côte d'Ivoire.

\subsubsection{Création des plateformes d'innovation banane plantain et évolution du nombre d'adhérents}

L'établissement des plateformes d'innovation banane plantain (PIP) a été rendu possible de septembre à décembre 2013 par l'action conjuguée du Ministère en charge de l'agriculture, du CNRA, de l'IITA, de l'ANADER et du FIRCA_WAAPP. La plateforme d'innovation YEBOYEKON d'Abengourou est la première en date (13 septembre 2013), puis ce fut successivement l'établissement de celle de N'DÈ N'FENIN-TÔH d'Agboville dans la région de l'Agneby Tiassa (25 septembre), de la PIP WOYÈ d'Adzopé (3 octobre), de la PIP d'Issia (5 décembre) et celle de Soubré dans la région de la Nawa (17 décembre 2013). Cette action marque le début de l'organisation et de la restructuration locale de la filière.

Ainsi, depuis leur création (2013), il ressort une diversité d'acteurs variant par zone d'implémentation et par parties prenantes. En effet, ces PIP sont composées d'acteurs directs de la chaîne de valeur (producteurs, pépiniéristes, commerçants et transformateurs) et des autres acteurs indirects ne possedant pas le produit, mais dont l'implication est nécessaire pour la commercialisation (transporteurs), les laisser-passés (forces de l'ordre), l'octroi de crédits (IMF), l'organisation des producteurs (ANADER), etc. Ces plateformes ont pour objet d'intégrer les différents maillons de la filière dans la mise en œuvre des changements (techniques, organisationnels) nécessaires à la diffusion de nouveaux cultivars.

Le nombre de groupements de producteurs impliqués variait entre 2 et 10 par PIP avec un nombre moyen d'adhérents allant de 150 jusqu'à 600 pour la plateforme de Soubré, qui compterait à ce jour environ 20 groupements. Ce nombre semble avoir évolué de 35 à 68 entre 2013 et 2016, ce qui traduit l'intérêt que porte cette catégorie d'acteurs au succès de la PIP. Ces groupements d'environ 1500 adhérents au total (FIRCA 2015), recensent près de $40 \%$ à $65 \%$ de femmes productrices de banane plantain, atteignant souvent environ 150 femmes par PIP. Ce qui pourrait se justifier par la présence d'au moins deux groupements de femmes productrices de vivriers par PIP en Côte d'Ivoire. Tous ces producteurs sont regroupés au sein d'une OPA. Par ailleurs, l'activité de pépiniériste s'est rapidement développée, comme c'est présentement le cas dans la PIP d'Agboville. Ces pépiniéristes assurent aux producteurs de banane plantain une pérennisation des plants améliorés et leur fourniture durable. 
Quant aux associations regroupant les commerçants, il en existe une voire deux par plateforme, avec au nombre des adhérents une majorité de femmes ( 80 à $90 \%$ ). À ce niveau, il serait judicieux de souligner que pour ce qui concerne les associations de commerçants du vivrier, les acteurs dans certains cas sont des producteurs-commerçants. Étant majoritairement détenues par des femmes, ces associations sont dénommées « coopératives de commerçantes du vivrier ». Certaines sont regroupées en fédération de coopératives (FENACOVICI, COCOVICO, CNAVICI) ${ }^{3}$, tandis que d'autres par contre ne seraient affiliées à aucune coordination. Cet état de fait, rendrait probablement plus difficile l'appropriation de la PIP par cette catégorie d'acteurs de la filière, de même que pour les restauratrices.

En plus de ces acteurs directs, une plateforme peut compter parfois en son sein un syndicat de transporteurs de banane plantain au nombre de 5 à 15 adhérents, ainsi qu'une institution de microfinance (IMF) ou une banque commerciale, de même qu'une filiale de revendeurs de produits phytosanitaires de 1 à 5 membres. Il faut noter également la présence dans certaines PIP de chefs coutumiers et de comités consultatifs dans les localités (cas de la ville d'Issia par exemple). Nos enquêtes ont également révélées l'introduction de certains acteurs de la vie civile et communautaire dans l'organisation de certaines PIP. Il s'agit du conseil général, des forces de l'ordre, de la mairie et du préfet de région comme c'est actuellement le cas de la PIP YEBOYEKON de la région d'Abengourou.

\subsubsection{Création des plateformes d'innovation banane plantain et évolution du nombre d'adhérents}

Trois années après son établissement, la perception du rôle de la PIP varie d'une région à l'autre et diffère de celle des institutions de recherche et des services d'intermédiation. En effet, pour certains présidents de plateforme, la PIP est une centrale d'achat tandis que pour d'autres, il s'agit d'un cadre réglementaire où tous les groupes d'acteurs de la filière devraient se rencontrer pour réaliser des échanges en tissant une solide relation d'affaire entre eux.

Pour les opérateurs économiques qui la composent, la PIP apparait comme : "un cadre de concertation, d'échanges et de partage entre les différents acteurs (directs et indirects) d'une même chaîne de valeur $(\mathrm{CdV})$; afin de résoudre leurs problèmes de production, de transformation, de commercialisation, de transport et de financement. Ce dispositif devrait permettre de défendre leurs intérêts communs à travers la recherche d'une plus-value partagée qui améliorerait leurs revenus et leurs moyens de subsistance et par conséquent leur condition de vie et leur bien-être » (FIRCA 2015).

Les institutions partenaires (IITA) et les structures de recherche (CNRA) conçoivent la PIP comme un outil de diffusion de nouvelles technologies (innovations et connaissances) et un dispositif de renforcement des capacités des bénéficiaires. Elle constitue donc un outil adéquat pour apporter des solutions aux contraintes de la majorité des acteurs d'une CdV et un outil pour la gestion du processus multi-acteurs dans cette chaîne. Il ressort également que la PIP représente surtout un moyen ou un cadre d'échanges pour améliorer l'identification des besoins des agriculteurs et diffuser de nouvelles technologies en milieu rural. C'est aussi un dispositif où travaillent ensemble plusieurs catégories d'acteurs afin de créer de la valeur en vue d'un partage équitable.

Le fonctionnement de ces plateformes s'est fait en 2 phases. Après leur mise en place, des bureaux exécutifs $(\mathrm{BE})$ se sont constitués à l'image des premiers acteurs directs (groupements de producteurs) qui avaient bénéficié des plants améliorés et des séances de formations sur les nouvelles pratiques culturales induites par l'usage des nouvelles variétés d'hybrides en 2012. Ainsi, le nombre et les catégories d'acteurs impliqués dans le bureau exécutif $(\mathrm{BE})$ différaient d'une plateforme à l'autre, mais chaque $\mathrm{BE}$ restait dans l'ensemble majoritairement dominé par les producteurs avec la présence de quelques commerçants, de transformateurs et de transporteurs.

\footnotetext{
${ }^{3}$ FENACOVICI : Fédération Nationale des Coopératives de Vivriers de Côte d'Ivoire ; COCOVICO : Coopérative des commerçants de vivriers de Cocody ; CNAVICI : Coordination Nationale des Acteurs du Vivrier de Côté d'Ivoire.
} 
En mai 2015 les institutions de recherches (IITA, CNRA) ont suggéré une nouvelle forme d'organisation des PIP avec un maximum théorique de 7 membres dans chaque $\mathrm{BE}$, soit en moyenne un représentant par groupe d'acteurs y compris le président de la PIP. En outre, les fréquences des rencontres du BE dans les PIP demeurent mensuelles, tandis que les assemblées générales (AG) sont convoquées de façon trimestrielle. Ces AG sont constituées par deux représentants de chaque groupement de producteurs, de coopératives de commerçants et d'associations de transformateurs ou de restauratrices, ayant adhéré et à jour de leur cotisation, ainsi que de chaque représentant des acteurs indirects membres de la PIP.

L'organisation et la motivation des acteurs demeurent cependant un enjeu majeur pour la survie de chaque PIP. Elle s'autofinance en effet, par les droits adhésion des groupements et associations et par les cotisations des membres. Toutefois, si cet autofinancement a eu lieu en 2014, il a été partiel en 2015 et reste hypothétique en 2016, au moment de nos investigations sur le terrain. Les montants recueillis sont versés dans un compte bancaire logé dans une institution financière habilitée située dans la région où est implantée chaque PIP, ce qui favorise également la constitution de leurs dossiers pour une formalisation légale comme c'est le cas actuellement pour les PIP d'Adzopé, d'Agboville et de Soubré.

\section{Cadrage conceptuel et méthodologique}

\subsection{SSI : Cadre d'analyse des changements de trajectoires technologiques basé sur les stratégies d'acteurs dans les filières agroalimentaires}

Le cadre conceptuel des systèmes d'innovation (SI) (LUNDVALL 1992), (HALL 2005), (TOUZARD et al., 2015) a été utilisé afin de caractériser le fonctionnement des plateformes d'innovation (HEKKERT et al., 2007) banane plantain (PIP) à travers la description des composantes, des acteurs et de leurs interactions (RÖLING 2009). Ce cadrage conceptuel a permis d'identifier dans notre étude quatre composantes du SSIA dans la filière banane plantain afin d'analyser la dynamique des interactions entre catégories d'acteurs et leurs stratégies de coordination.

Ce SSIA se compose ainsi des principales parties prenantes suivantes: (i) la recherche, (ii) les services d'intermédiation (vulgarisation et conseil), (iii) les professionnels ou acteurs économiques constitués des acteurs de l'agrofourniture, de producteurs, de commerçants et de transformateurs, et enfin (iv) les institutions de financement de l'innovation agricole dans cette filière banane plantain.

Cette étape devrait aboutir à l'évaluation des contraintes au fonctionnement de ce système d'innovation agricole (SIA) encore en construction dans le secteur banane plantain en Côte d'Ivoire.

La caractérisation du fonctionnement des PIP contribuerait enfin à identifier les arrangements et changements institutionnels (HOUNKONNOU et al., 2012) entre catégories d'acteurs, tout en intégrant la diversité des contextes socio-culturels dans lesquels évoluent ces PIP.

Cette démarche permet de mettre en relief les mécanismes de coévolution des dimensions de l'innovation: "hardware, software et orgware" (KLERKX et al., 2012), que sous-tendraient les processus d'innovation sur la banane plantain.

\subsection{Conceptualisation des quatre composantes d'un Système Sectoriel d'Innovation Agricole}

Le système sectoriel d'innovation agricole (SSIA) sur la banane plantain que nous identifions dans le contexte ivoirien, est un dispositif qui met en relation quatre composantes qui structurent les innovations socio-technologiques en cours, à savoir : (1) la composante recherche, (2) la composante intermédiation, (3) la composante chaîne de valeur et (4) la composante financement de l'innovation (figure 2.2). Ces éléments agissent et interagissent dans ce système agroalimentaire pour la création de biens et / ou de services. 


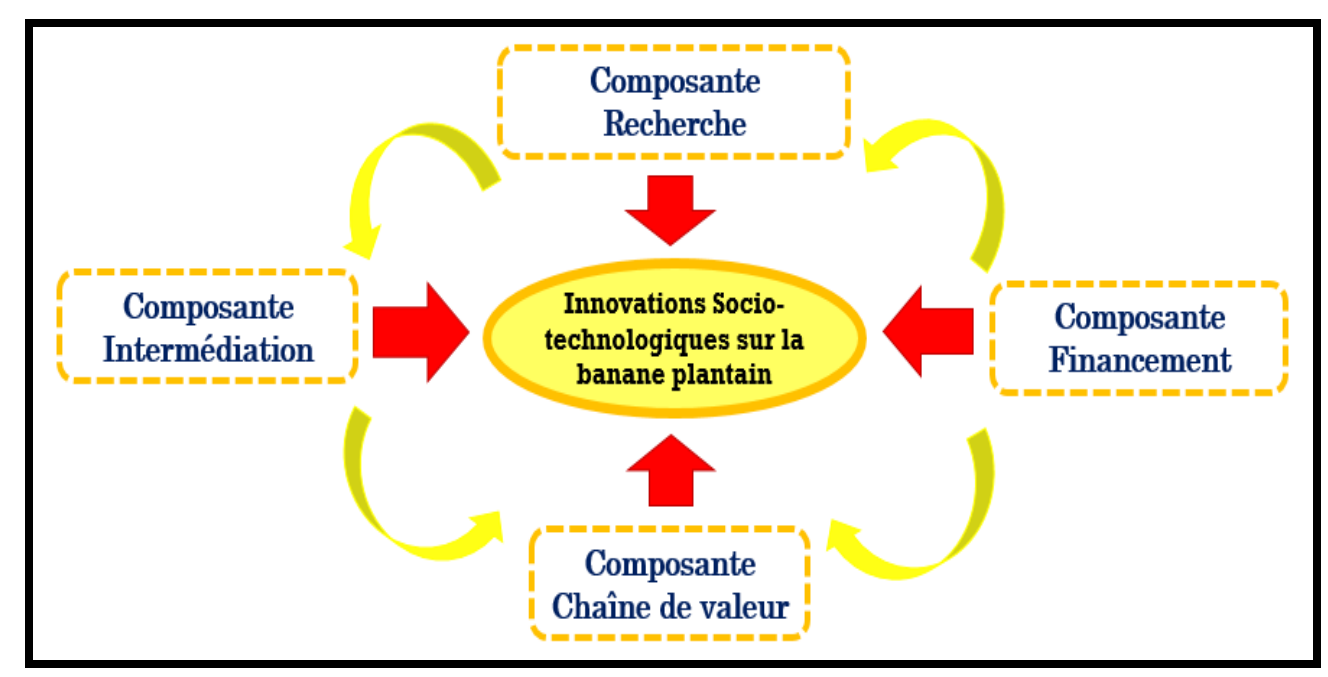

Figure 2.2. Le SSIA dans la filière banane plantain en Côte d'Ivoire ${ }^{4}$ - source : auteur

La "composante recherche", en produisant des connaissances de base, des technologies et des produits divers, oriente les processus d'innovation dans le secteur vivrier (MALERBA 2005). En Côte d'Ivoire, cette action est dévolue dans le domaine agricole par les institutions des recherches nationales (CNRA, I2T, CNS BP), les universités et grandes écoles d'ingénieurs et de techniciens supérieurs (INPHB, ENSEA, UFHB, UNA), et les organismes de recherche et de coopération sous régionaux (CARBAP) et internationale (CIRAD, IITA, CGIAR).

Pour assurer la circulation des connaissances produites par la recherche auprès des professionnels ou acteurs directs dans les chaînes de valeur $(\mathrm{CdV})$, une "composante intermédiation" a été également identifiée. Les acteurs intermédiaires de l'innovation encore nommés "brokers » (KLERKX et al., 2012), (KILELU et al., 2013), ont la spécificité de rendre plus accessibles les connaissances théoriques en les transformant en langage plus adéquat pour en assurer par la suite la diffusion ou le transfert de technologies aux acteurs directs de la $\mathrm{CdV}$ ou aux bénéficiaires des innovations. Les structures au sein de cette composante sont en partie détenues par l'État. Elles assurent l'encadrement, la formation, le renforcement de capacité, la vulgarisation ainsi que le conseil sur les pratiques agricoles dans ce secteur sur lequel repose toute l'économie ivoirienne: l'agriculture. Il s'agit de l'ANADER, l'INADES Formation, l'ADCVI, les ONGs, les OPA, certaines coopératives agricoles et groupmenents villageois, les Ministères en charge de l'agriculture, de l'économie et des finances, de l'industrie, de l'artisanal et des PME.

Les professionnels ou entrepreneurs privés encore appelés acteurs directs ou indirects de la $\mathrm{CdV}$, exerçant dans la filière banane plantain, ont été tous regroupés dans ce SSIA au niveau de la "composante chaîne de valeur". Au sein de cette composante, les agents économiques certes hétérogènes, bénéficient de formation, d'apprentissage, de renforcement de leur capacité pour les techniques de production, de commercialisation et de transformation, et également de soutiens (matériels et / ou financiers). Cette composante regroupe des acteurs économiques qui développent généralement des relations marchandes dans le but de réaliser des profits. Elle regroupe autant les acteurs de l'amont de la filière que ceux de l'aval. Au centre de cette composante CdV se trouve les producteurs de banane plantain comprenant aussi bien des hommes que des femmes, mais beaucoup plus de femmes du secteur vivrier en général. Les acteurs de l'amont assurent le service d'agrofourniture d'intrants tels que les pépiniéristes et les fournisseurs de produits phytosanitaires. À l'aval de la $\mathrm{Cdv}$, on note surtout la présente de transporteurs, de commerçants, de transformateurs (indutriels et artisanaux) et d'acteurs de la grande et moyenne distribution avec des produits bruts ou transformés (farine, patisserie, chips de banane plantain) destinés soit aux marchés locaux ou soit à

\footnotetext{
${ }^{4}$ Graphique de Euphrasie C. M. Angbo-Kouakou, 2016 (à paraître dans sa Thèse de Doctorat)
} 
l'exportation vers les pays de la sous-région ouest africaine (Burkina Faso, Mali, etc.) ou plus encore à destination de certains pays de l'Union Européenne et d'Amérique.

La "composante financement" de l'innovation quant à elle soutient les activités de recherche, de développement, d'innovations technologiques et de vulgarisation des connaissances d'une part et d'autre part, réalise l'appui au secteur agricole. Elle est constituée par les bailleurs de fonds à l'international (Banque mondiale), les banques régionales (BAD, BCEAO, BOAD), et les banques commerciales nationales (BNI, BICICI, SIB) ainsi que le FIRCA au niveau national. Les institutions locales de microfinance (IMF) contribuent également à l'octroi de microcrédits et au developpment de l'épargne telles que la Mutuelle d'octroi de crédits aux femmes de la PIP d'Agboville (MUCREF) et l'Institution Financière d'Epargne et de Crédit des Coopérateurs (IFECC) de la PIP de la Nawa à Soubré. Seules ces deux structures de microfinances entretiennent à ce jour des relations de support financier avec les PIP.

En plus des relations intragroupes formelles, s'opère des interactions informelles entre composantes, ce qui confère le sens de système à ce dispositif sectoriel. Ces interactions entre principaux acteurs et parties prenantes de ces composantes se réalisent fréquemment dans le cadre de la mise en œuvre d'une politique publique, d'un programme ou projet de développement national.

Une application de ce SSIA à la nouvelle politique de réduction de la dépendance alimentaire en Côte d'Ivoire serait assurément bénéfique pour obtenir un impact positif de ce programme d'amélioration de la productivité agricole (WAAPP) sur l'atteinte des enjeux de sécurité alimentaire.

\section{IMPORTANT.}

Le SSIA appliqué aux stratégies d'interaction des acteurs de la filière banane plantain en Côte d'Ivoire sera décrit à travers ses 4 composantes et sa relation avec le dispositif expérimentale dénommé Plateforme d'Innovation Banane Plantain mis en œuvre par le WAAPP, abrégé dans cette étude par PIP.

\subsection{Cadre méthodologique et analytique}

Dans le cadre de la collecte de données primaires et pour les besoins de cette étude, ce sont le Ministère de tutelle de ce programme d'amélioration de la productivité (MINADER), l'organe exécutif (FIRCA_ WAAPP), le centre de coordination des programmes de recherche sur la banane plantain (CNRA) et l'agence technique de vulgarisation (ANADER), qui ont été sélectionnés pour mener des entretiens semi-directifs. Des enquêtes à dires d'experts ont été également réalisées en France (VITROPIC, CIRAD et Experts) et en Côte d'Ivoire (Chercheurs et Experts) en 2015 et 2016 afin de mieux comprendre et de décrire le processus d'innovation socio-technologique en cours dans la filière banane plantain ivoirienne.

Une mission d'enquêtes de terrain a été par ailleurs réalisée dans quatre PIP sur les cinq, du 25 au 30 Avril 2016. Ces enquêtes ont été rendues possibles par l'usage de guides d'entretien semi-directif administrés en face à face. Les entretiens ont été réalisés au siège de chaque bureau exécutif (BE) de la PIP situé pour la plupart dans les locaux des directions départementaux ou régionaux de l'ANADER à Soubré, Agboville, Adzopé et Abengourou. Les collectes se sont déroulées auprès des présidents élus de chaque PIP avec la participation en plus d'un membre ou deux de chaque BE, soit un total de 12 acteurs directs, composés de producteurs, pépiniéristes, commerçantes et restauratrices membres de la PIP, parfois en présence de l'agent conseil de l'ANADER en charge de la mise en œuvre du projet WAAPP.

Le tableau 1 ci-après présente un résumé des méthodes d'enquêtes utilisées et la nature des informations collectées. Ces enquêtes ont servi à une meilleure compréhension de l'organisation de ce SSIA et à l'analyse de son évolution. 


\begin{tabular}{|c|c|c|c|c|c|c|}
\hline \multirow[b]{2}{*}{ Structures } & \multirow[b]{2}{*}{$\begin{array}{l}\text { Dates de } \\
\text { collecte }\end{array}$} & \multicolumn{4}{|c|}{ Méthodes de Collecte } & \multirow[b]{2}{*}{ Informations recherchées } \\
\hline & & $\begin{array}{c}\text { Réunions } \\
\text { Bilan }\end{array}$ & $\begin{array}{l}\text { Entretiens } \\
\text { Semi- } \\
\text { directifs }\end{array}$ & $\begin{array}{l}\text { Entretiens } \\
\text { non } \\
\text { Structurés }\end{array}$ & $\begin{array}{l}\text { Focus } \\
\text { groupe }\end{array}$ & \\
\hline $\begin{array}{l}\text { MINADER } \\
\text { (Ministère en }\end{array}$ & $\begin{array}{l}\text { Décembre } \\
2014\end{array}$ & 1 & & & & $\begin{array}{l}\text {-État de l'art sur la filière banane } \\
\text { plantain en Côte d'Ivoire } \\
\text {-Interaction entre MINADER, } \\
\text { ANADER, OCPV et GIZ } \\
\text {-Évolution du contexte : production et } \\
\text { commercialisation } \\
\text {-Partenariat entre le MINADER et le } \\
\text { Programme WAAPP pour réduire la } \\
\text { dénendance alimentaire }\end{array}$ \\
\hline $\begin{array}{c}\text { charge de } \\
\text { l'Agriculture) }\end{array}$ & Juin 2015 & & 1 & & & $\begin{array}{l}\text {-Historique des choix et introduction } \\
\text { des variétés d'hybrides améliorées et } \\
\text { résistantes : Pita 3, Fhia 21, Big } \\
\text { Ebanga } \\
\text {-Perception du rôle des Plateformes } \\
\text { d'Innovation Plantain } \\
\text {-Fonctionnalité des systèmes } \\
\text { d'innovation agricole (AIS) }\end{array}$ \\
\hline $\begin{array}{l}\text { FIRCA_WAAPP } \\
\text { (Organe exécutif }\end{array}$ & Juin 2015 & & 2 & 2 & 1 & $\begin{array}{l}\text {-Perception du rôle des Plateformes } \\
\text { d'Innovation Plantain } \\
\text {-Identification des membres du réseau } \\
\text { d'acteurs dans la PIP } \\
\text {-Interaction entre professionnel et } \\
\text { institution de recherche } \\
\text {-Interaction entre professionnel et }\end{array}$ \\
\hline du WAAPP en CI) & $\begin{array}{l}\text { Avril } \\
2016\end{array}$ & 1 & & & & $\begin{array}{l}\text {-Identification du rôle des membres } \\
\text { de la PIP et les bénéfices } \\
\text {-Intervention du FIRCA avant la } \\
\text { création des PIPs } \\
\text {-Enjeux de productivité agricole et la } \\
\text { place du FIRCA }\end{array}$ \\
\hline $\begin{array}{l}\text { VITROPIC } \\
\text { (Fournisseurs de } \\
\text { Vitro Plants) }\end{array}$ & $\begin{array}{l}\text { Mars } \\
2016\end{array}$ & & & 1 & & $\begin{array}{l}\text {-Information sur les services de } \\
\text { l'entreprise, fournis en CI } \\
\text {-Relations de l'entreprise avec le } \\
\text { Programme WAAPP_CI } \\
\text {-Enjeux de la collaboration de cette } \\
\text { structure avec le FIRCA } \\
\text {-Informations sur les enjeux de la } \\
\text { production de plantain }\end{array}$ \\
\hline $\begin{array}{c}\text { Plateformes } \\
\text { d'Innovation } \\
\text { Plantain (PIP) }\end{array}$ & $\begin{array}{l}\text { Avril } \\
2016\end{array}$ & & 4 & & & $\begin{array}{l}\text {-Perception du rôle des Plateformes } \\
\text { d'Innovation Plantain } \\
\text {-Identification des membres du réseau } \\
\text { d'acteurs dans la PIP } \\
\text {-Interaction entre professionnel et } \\
\text { structure d'appui conseil } \\
\text {-Interaction entre professionnel et } \\
\text { institution de recherche } \\
\text {-Identification du rôle des membres } \\
\text { de la PIP et les bénéfices }\end{array}$ \\
\hline
\end{tabular}




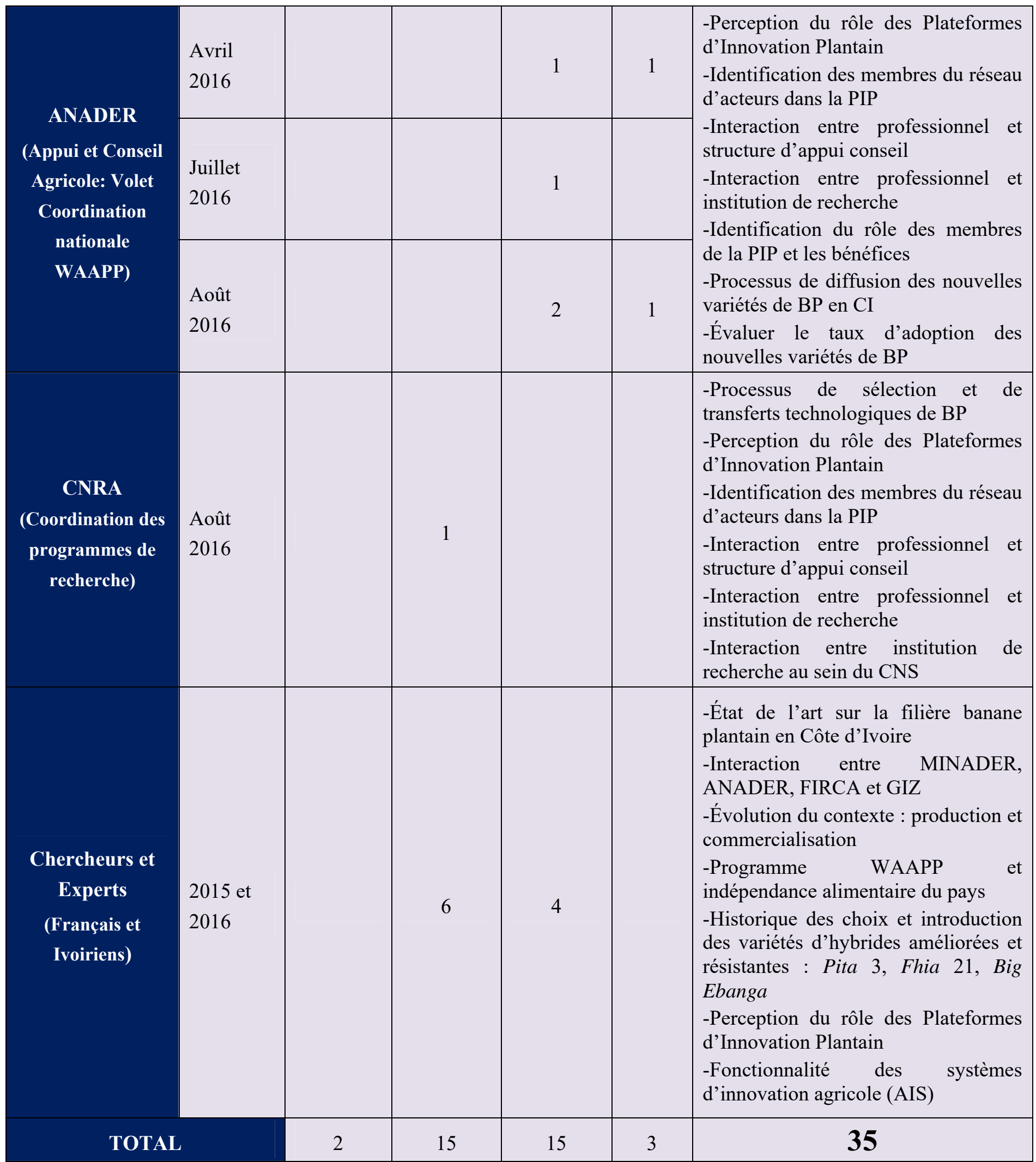

Tableau 2.3. Collectes de données - Source : auteur 


\section{Résultats}

\subsection{Fonctionnalité des plateformes d'innovation banane plantain de Côte d'Ivoire}

La PIP est un outil multifonctionnel ayant deux orientations pour les opérateurs économiques de cette filière agricole. La première est de faire interagir les acteurs qui pilotent l'innovation (intermédiaires, chercheurs...) avec les acteurs des CdV (producteurs, commerçants, transformateurs) à partir des résultats de la recherche (CNRA, IITA, CNS BP). Le processus de ce transfert technologique se réalise principalement par: (i) la sélection et l'introduction par la recherche de deux hybrides améliorés, (ii) la diffusion de ces nouvelles technologies (innovations et connaissances) au sein des groupements de producteurs et (iii) la formation, l'apprentissage et le renforcement des capacités des acteurs sur l'utilisation du paquet technique 5 associé à cette technologie.

La seconde orientation des PIP est de restructurer les coordinations entre les acteurs des CdV pour accélérer l'usage ou non des propositions techniques de départ (variétales) à l'aide d'une gestion du processus multi parties prenantes en leur sein. Les enquêtes révèlent plusieurs contraintes de gouvernance et d'efficacité des PIP dont rendent comptent la dilution des AG même si les réunions mensuelles de bureaux exécutifs sont tenues régulièrement. Des problèmes de fonctionnement classiques liés au manque de moyens de transport pour les membres du BE ont pénalisé fortement les tâches qui leurs sont dévolues auprès des producteurs et adhérents, comme c'est le cas dans la PIP de la Nawa à Soubré.

\subsection{Réorganisation des composantes du SSIA par les Plateformes}

La réorganisation par les plateformes des quatre composantes du SSI sur la banane plantain a pour conséquence l'organisation et la coordination des activités des groupes d'acteurs impliqués dans la diffusion des nouvelles technologiques. Cette organisation se situe en effet à plusieurs niveaux : (1) analyse du choix des nouvelles variétés introduites dans chaque localité ; (2) prise en compte de leurs conditions d'acceptabilité par les bénéficiaires; (3) nécessité de l'intégration des processus d'innovation endogènes réalisés par les acteurs concernés; (4) réorientation des trajectoires technologiques par les politiques publiques introduites dans la filière ; (5) émergence des arrangements institutionnels entre acteurs membres de la plateforme; (6) identification des besoins réels des acteurs dans des contextes hétérogènes (diversité des zones de production agricole du plantain) ; et par-delà, (7) prise en compte des préférences alimentaires des consommateurs potentiels en général et celles des consommateurs locaux plus particulièrement.

Sur la coordination des activités des groupes d'acteurs hétérogènes, trois relations entre parties prenantes de la filière sont apparues les plus significatives sur le terrain au cours de l'expérimentation variétale du projet WAAPP. Ce sont (i) la collaboration entre les structures de recherche \& développement et les agences d'intermédiations ou services d'encadrement des producteurs en milieu rural, (ii) les interactions entre ces structures d'encadrement et les groupements de producteurs bénéficiaires de l'innovation, et enfin (iii) les échanges entre les structures de recherche et les professionnels de la $\mathrm{CdV}$ pour une meilleure orientation des politiques publiques dans le choix des cultivars.

\footnotetext{
${ }^{5}$ Le paquet technique proposé par l'ANADER en 2012 aux groupements de producteurs était composé des variétés Pita 3 et Fhia 21 , d'engrais chimique (urée et / ou NPK), de pesticides (Furadan), des nouvelles techniques culturales dispensées à partir de formations théoriques et appliquées dans les champs écoles, suivis du transfert de compétences sur des parcelles individuelles en monoculture.
} 
C'est ainsi que les PIP ont fait interagir les composantes du SSI en 2014 et organisé une construction participative des conditions d'adoption locales des hybrides selectionnées depuis 2007 (figure 3.2). Cependant la diffusion nationale qui avait suivi en 2012 n'intègre pas la prise en compte des besoins liés à la diversité des contextes et des régions, encore moins les préférences des consommateurs locaux (JIGGINS, HOUNKONNOU, SAKYI-DAWSON, KOSSOU, TRAORÉ, RÖLING et VAN HUIS 2016).

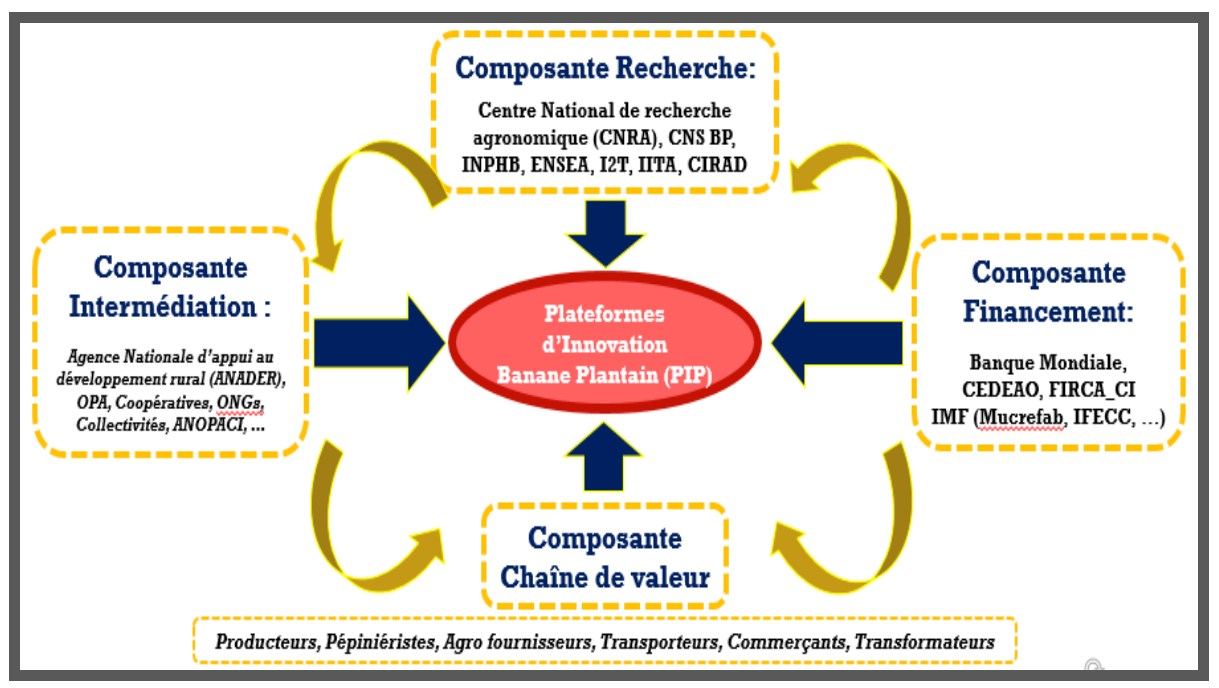

Figure 3.2. Structuration des Plateformes d'Innovation Banane Plantain (PIP).

\subsection{Réorientation des trajectoires technologiques dans la filière banane plantain en Côte d'Ivoire}

La réorientation des décisions des politiques publiques a été rendue possible par les échanges permanents entre plusieurs catégories d'acteurs et parties prenantes de la filière au sein de la composante chaîne de valeur. Elle a eu lieu grâce aussi aux interactions multiples développées entre les différentes composantes (professionnels, intermédiaires, institutions de recherche et organismes de financement) de ce SSIA. C'est ainsi que les PIP ont fait émerger des dynamiques de coordination des acteurs de diverses régions permettant de spécifier les besoins d'amélioration de la productivité des plantations et de répondre aux préférences alimentaires des consommateurs locaux de banane plantain.

Après la diffusion en 2012 des variétés PITA 3 et FHIA 21 et le transfert des technologies culturales liées à l'usage de ces variétés, par l'ANADER, opéré sous l'impulsion de la recherche agronomique, l'établissement participatif en 2013 de la PIP dans les principales zones de production a favorisé l'identification de cultivars de bananiers plantains proches des caractéristiques des principales variétés locales (Agnrin et Affôtô). Ainsi, les interactions entre acteurs de la filière au sein des PIP ont permis d'exprimer les besoins des producteurs et consommateurs locaux en matière du choix du matériel végétal pour le bananier plantain.

De façon unilatérale, le FIRCA_WAAPP a changé par ailleurs de politiques d'innovation en introduisant par la suite la variété Big Ebanga en 2014, un cultivar originaire du Cameroun.

\section{Illustration de la gouvernance des PIP :}

\section{Cas de la PIP N'DÈ N'FENIN-TOH de l'Agneby Tiassa}

Pour illustrer nos propos, nous avons choisi de mettre en exergue le cas de la gouvernance de la PIP d'Agboville comme un modèle de réussite de ces dispositifs expérimentaux. 
À Agboville par exemple, cette capacité institutionnelle théorique est en deçà en mai 2016 avec 5 membres (1 producteur, 1 pépiniériste, 1 commerçant, 1 structure de transformation de Pita 3 en Chips, 1 représentant des transporteurs), tandis qu'à Soubré, elle a été largement atteinte avec 10 acteurs (2 producteurs, 2 commerçants, 2 transformateurs, 1 vendeur de produits phytosanitaires, 2 représentants de la Microfinance, 1 représentant du CNRA), ce qui pourrait dénoter de l'intérêt affiché du président de la PIP de Soubré d'en faire une centrale d'achat, au-delà d'un simple cadre de concertation entre acteurs. Par ailleurs, l'assemblée générale de la PIP d'Agboville est constituée de 2 représentants par groupe d'acteurs impliqués dans la PIP en 2013, soit 20 membres pour les 10 groupements de producteurs, 4 membres pour les 2 associations de commerçants et 2 membres pour le syndicat de transporteurs.

Par la suite 5 groupements de producteurs y ont intégré (10 membres), de même qu'une unité industrielle de transformation du PITA 3 en Chips, ainsi que 2 pépiniéristes. Toutefois, cette PIP ne compte pas de vendeurs de produits phytosanitaires car les parcelles ne nécessitaient pas d'apports d'engrais ou de pesticides lors des tests d'expérimentation (FAO, 2011). Par contre, pour lutter contre les maladies du bananier plantain, certains producteurs d'Agboville utilisaient de la cendre, tout comme ceux des PIPs d'Adzopé et d'Abengourou. Toutefois, ce BE compte plus d'hommes que de femmes, contrairement à la configuration des groupements de producteurs de banane plantain de la région (Koffi, 2008). Cette situation s'expliquerait par le fait que plusieurs femmes des groupements de producteurs de plantain de l'Agneby Tiassa ignorent l'existence même de la PIP dans leur région d'autant plus que la plupart du temps c'est le service d'intermédiation (ANADER) qui assure la coordination des activités réalisées dans le cadre de la PIP, et surtout au nom du projet WAAPP.

\section{Encadré 3.3. Gouvernance de la PIP d'Agboville}

\section{Mise en discussion des fonctionnalités du système et des arrangements institutionnels}

\subsection{Fonctionnalités du SSI agricole pour la filière banane plantain en Côte d'Ivoire}

Le renouvellement des trajectoires technologiques d'innovation change la structuration du SSI dans la filière banane plantain. Ainsi les fonctionnalités initiales (cf. 2.2) de ce SSI s'en trouvent modifiées (HEKKERT et al., 2007). De fait, le rôle des SSIA est (i) de freiner ou (ii) de favoriser la diffusion d'une technologie suite à une meilleure compréhension des besoins locaux. Ce changement traduit un basculement du modèle d'innovation diffusionniste fondée sur l'introduction d'hybrides qui passe de «technologies pools» à un processus «buttom-up» favorisant l'adaptation de technologies introduites.

Dans la première étape de la sélection participative en 2007 qui a précédé la diffusion massive des hybrides PITA 3 et FHIA 21 au sein des groupements de producteurs en 2012, la politique d'innovation était principalement de type «technologie pool », associant une conception « top down » caractérisée par un transfert de technologies identifiées par la recherche et vulgarisées par les «brokers» à travers des séances de formation, d'apprentissage et de renforcement de capacités des bénéficiaires de ces innovations, en lien avec la dimension "software" de l'innovation.

Cette approche qui a prévalu avant l'établissement des plateformes a conduit selon les responsables des PIP d'Adzopé et de Soubré, à un refus généralisé de l'hybride PITA 3 par les groupements de producteurs bénéficiaires, dans certaines localités, et à une acceptation mitigée de l'hybride FHIA 21. Cette situation a milité en faveur de l'identification des besoins réels de productivités des producteurs et des préférences des consommateurs locaux à travers une conception «buttom-up » par interactions entre toutes les quatre composantes du SSIA au sein des PIP. 
Dans un second temps, elle a structuré une politique d'innovation fondée sur l'introduction du Big Ebanga, variété traditionnelle cultivée au Cameroun, mais non issue de la création variétale portée par la recherche agronomique en Côte d'Ivoire.

\subsection{Arrangements et Changements institutionnel}

L'utilisation des plateformes comme moyen d'arrangements ou de changements institutionnels (HOUNKONNOU et al., 2012) met en exergue l'interaction entre les différentes composantes des SSI et la coévolution des trois dimensions de l'innovation (hardware, software et orgware), et le cas échéant, de manière spécifique la dimension "orgware". De fait, les Arrangements Institutionnels (AI) entre acteurs se sont renforcés entre les groupements de producteurs et les pépiniéristes formés aux méthodes de sélection et de multiplication végétales. Ces nouveaux AI informels se matérialisent par des échanges à moindre coûts du matériel végétal (vitro plants ou vivo plants) entre ces deux groupes d'acteurs. Ainsi, le programme d'amélioration de la productivité (WAAPP) a renforcé la professionnalisation du métier de pépiniériste sur le bananier plantain en Côte d'Ivoire depuis 2015.

L'objectif de favoriser un contact permanent entre les acteurs de la composante recherche et les producteurs sur la sélection a permis une meilleure orientation de la recherche agronomique pour mieux satisfaire les besoins de ces acteurs. Ainsi, plusieurs chercheurs rencontrés déclarent désormais revenir sur des travaux permettant d'accroitre les productivités de variétés locales. L'identification de ces variétés locales et leurs potentiels d'amélioration génétiques deviennent aujourd'hui l'objectif commun pour ces deux composantes de ce SSIA (recherche et chaîne de valeur).

Ces contextes ont également permis de montrer que la diffusion à grande échelle des nouvelles variétés dites améliorées a certes ponctuellement répondu à des besoins d'expérimentation et de disponibilité de matériels de plantation. Mais la faible maitrise des techniques culturales pendant la récolte a ensuite augmenté les pertes post-récoltes et détérioré les relations entre producteurs et acheteurs (commerçants et consommateurs). Tous les acteurs enquêtés (chercheurs, producteurs...) sont dès lors confrontés de manière simultanée à la difficulté de devoir trouver de nouvelles techniques de cuissons et de préparations alimentaires pour la consommation des hybrides introduits.

\subsection{Renouvellement des processus d'innovation technologiques}

Les deux hybrides introduites PITA 3 et FHIA 21 qualifiées de plants améliorées de bananiers plantains à haut rendement et potentiellement tolérant à la Cercosporiose ont fait leur preuve dans les plantations de cultures vivrières en Côte d'Ivoire, caractérisés par un rendement plus élevé que les variétés anciennes. Mais cela ne répond pas necessairement aux besoins en nouvelles variétés de plants pour les producteurs locaux et aux préférences alimentaires des consommateurs ivoiriens (LOPEZNICOLAS et MERONO-CERDAN 2011). Aussi, le rejet de ces variétés d'hybrides selon les régions d'expérimentation, est bien sûr variable selon les habitudes alimentaires des groupes de populations.

Cette réalité locale se traduit par les propos des présidents des PIP d'Agboville et d'Adzopé : « Chez nous ici à Agboville les deux variétés ont majoritairement été accepté par la population locale puisque nous mangeons la banane plantain verte (non mure)» (M. Ado Joseph). "Dans nos habitudes alimentaires nous les Attié d'Adzopé nous préférons toujours la banane plantain bien mure et jaune, par contre les nouvelles variétés de PITA ne jaunisse jamais, ce qui nous a rendu la tâche très difficile pour la commercialisation et la consommation locale » (M. Achy Jean Sylvio).

De fait, le travail réalisé permet de rendre compte que l'objectif d'introduire des hybrides pour intensifier les rendements sur le bananier plantain était plus pour la transformation industrielle en chips ou la pâtisserie, et à des fin d'exportation, que celui de répondre aux besoins de la consommation en frais de la population. C'est ce qu'explique le président de la PIP M. Achy : « À Adzopé, nous avions accepté de poursuivre la production de PITA pour la vendre à la nouvelle unité de transformation de la banane plantain en Chips ». Selon Dame Nemeu Déborah du bureau exécutif de la PIP de Soubré : 
« Face à la mévente des nouvelles variétés de banane plantain, j'ai décidé d'en faire de la farine, ce qui m'a servi par la suite pour faire des gâteaux et m'a valu d'être filmée par le FIRCA_WAAPP à plusieurs reprises pour montrer mon exemple aux autres producteurs et transformateurs de banane plantain ».

Ainsi, répondre aux besoins locaux impliquerait alors de combiner les objectifs de production aux préférences alimentaires variables et diverses des ménages ruraux (DURY et BOCOUM 2012), dans une économie avec des us et coutumes très hétérogènes (GIBBON et PONTE 2005), (CARDENAS et CARPENTER 2008).

Les PIP ont fait donc remonter les préférences des professionnels de la chaîne de valeur marquant ainsi les insuffisances des transferts technologiques diffusionnistes dans les pays en developpement.

\section{Conclusion}

L'objectif de diffusion des variétés d'hybrides résistantes aux maladies et à hauts rendements de bananiers plantains en Côte d'Ivoire a généré la création de dispositifs expérimentaux : les Plateformes d'Innovation Plantain (PIP). Cette étude analyse l'incidence de quatre PIP sur la restructuration des Systèmes Sectoriels d'Innovation Agricole (SSIA) et la réorientation des trajectoires technologiques dans la filière banane plantain en Côte d'Ivoire.

Elle contribue ainsi à l'évaluation de l'incidence des plateformes d'innovation sur l'organisation des quatre composantes du SSIA sur la banane plantain (Recherche, Intermédiation, Chaîne de valeur et Financement) identifiées par la mise en complémentarité de plusieurs zones de production où sont respectivement implémentées ces PIP (Abengourou, Adzopé, Agboville, Soubré et Issia).

Il ressort de notre étude que c'est la coévolution entre ces quatre composantes du SSIA et les trois dimensions de l'innovation (hardware, software et orgware) qui restructure et influence les processus de décisions publiques dans la sélection et l'émergence de nouvelles innovations technologiques. La stratégie de développement de la filière banane plantain par l'introduction d'hybrides dites améliorées (PITA 3, FHIA 21) n'a pas vraiment réussie. En revanche elle a induit l'introduction de la variété Big Ebanga.

Les PIP ont par ailleurs réorienté et mis en cohérence les stratégies des acteurs (recherche, chaîne de valeur, intermédiation) permettant de prendre en compte les besoins d'amélioration variétaux émis par les producteurs en fonction des préférences alimentaires locales et non de celles des potentielles industries ou d'unités de transformations œuvrant pour l'exportation.

Ainsi, les PIP font émerger des dynamiques de coordination multi parties prenantes et contribuent au renforcement des capacités des acteurs de la filière à faire des choix techniques, ce qui a permis de restructurer le SSIA dans la filière banane plantain. Nos résultats interrogent cependant l'effectivité à venir de ces PIP en Côte d'Ivoire.

\section{Bibliographie}

ADEKUNLE A. A., FATUNBI A. O., « Approaches for setting-up multi-stakeholder platforms for agricultural research and development », World Applied Sciences Journal, 16 (7), 981-988, 2012.

BLONDEL S., BRIN R., KOFFI C., "Organisation des filières bananes ivoiriennes: Une étude de terrain expérimentale », Document de travail du GRANEM n 2014-03-042, Granem, Université d'Angers, septembre 2014. http://granem.univ-angers.fr/_attachments/cahiers-2014-article-3/DT_GRANEM_42.pdf?download=true 
BRICAS N., «Les marchés alimentaires urbains en Afriques de l'Ouest : Facteurs de dépendance ou d'entrainement de l'agriculture ? » dans Fok, M., Ndoye, O., \& Koné, S. (2015), AGRAR-2013: 1st conference of African research on agriculture, food, and nutrition. Yamoussoukro, Côte d'Ivoire, June 4-6, 2013. Agriculture and the challenges of food and nutrition in Africa: the contributions of research in the cotton zone. Presses Agronomiques de Gembloux. 2013.

CARDENAS J. C., CARPENTER J., «Behavioral development economics: Lessons from field labs in the developing world », The Journal of Development Studies, 44 (3), 311-338, 2008.

CGIAR RESEARCH PROGRAM (CRP) ON ROOTS, TUBERS AND BANANAS (RTB), « Tools for improved cropping systems intensification in mixed RTB systems with plantain in West and Central Africa », RTB Workshop Report, 10-14 November 2013, Abidjan, Côte d'Ivoire, 48 p, 2013.

CHATAIGNER, J., TANO, K., "L'économie de la banane plantain en Côte d'Ivoire », Cahiers ivoiriens de recherche économique et sociale (CIRES), (27), 31-102, 1980.

CHALEARD, J. L., Temps des villes, temps des vivres: l'essor du vivrier marchand en Côte d'Ivoire, Paris, KARTHALA Editions, Collection Hommes et Sociétés, 661 p, 1996.

CNRA “CENTRE NATIONAL DE RECHERCHE AGRONOMIQUE” DE COTE D'IVOIRE, Rapport annuel des activités de recherche de 2007, 77 p, 2008.

DURY S., BOCOUM I., «Le «paradoxe» de Sikasso (Mali): pourquoi «produire plus» ne suffit-il pas pour bien nourrir les enfants des familles d'agriculteurs? », Cahiers Agricultures, 21 (5), 324-336, 2012.

FIRCA_WAAPP "FONDS INTERPROFESSIONNEL POUR LA RECHERCHE ET LE CONSEIL AGRICOLE" DE COTE D'IVOIRE, Rapports de l'atelier bilan sur les Plateformes d'Innovation Plantain, Riz, Maïs et Manioc, Juin 2015.

GEREFFI G., HUMPHREY J., STURGEON T., « The governance of global value chains », 0, 12(1), 78-104, 2005.

GIBBON P., PONTE S., «Trading down: Africa, value chains, and the global economy », Temple University Press. Philadelphia (PA), 2005.

HALL A., « Capacity development for agricultural biotechnology in developing countries: an innovation systems view of what it is and how to develop it », Journal of international development, 17 (5), 611-630, 2005.

HEKKERT M. P., SUURS R. A., NEGRO S. O., KUHLMANN S., SMITS R. E., «Functions of innovation systems: A new approach for analyzing technological change », Technological forecasting and social change, 74 (4), 413-432, 2007.

HOUNKONNOU D., KOSSOU D., KUYPER T. W., LEEUWIS C., NEDERLOF E. S., RÖLING N., ..., VAN HUIS A., "An innovation systems approach to institutional change: smallholder development in West Africa », Agricultural systems, 108, 74-83, 2012.

HUGON P., «Filières agricoles et politique macroéconomique en Afrique Sub-Saharienne », Economie des politiques agricoles dans les pays en développement, Les aspects macro-économiques, Paris, Revue Française d'Economie, 83$121,1994$.

JIGGINS J., HOUNKONNOU D., SAKYI-DAWSON O., KOSSOU D., TRAORÉ M., RÖLING N., VAN HUIS A., «Innovation platforms and projects to support smallholder development-experiences from Sub-Saharan Africa », Cahiers Agricultures, 25, 2016.

KILELU C. W., KLERKX L., LEEUWIS, C., « Unravelling the role of innovation platforms in supporting co-evolution of innovation: contributions and tensions in a smallholder dairy development program », Agricultural systems, 118, $65-77,2013$.

KLERKX L., VAN MIERLO B., LEEUWIS, C., « Evolution of systems approaches to agricultural innovation: concepts, analysis and interventions », In Farming Systems Research into the 21st century: The new dynamic (pp. 457-483). Springer Netherlands, 2012.

KOFFI S. K., Enquête de référence sur la filière banane et plantain, Rapport Final, Station de recherche de Bimbresso, CNRA, 20 p, 2001.

KOFFI S. K., «Rôle des ressources génétiques dans l'essor du secteur bananier plantain en Côte d'Ivoire », Regional conference on Plant genetic resources and food security in West and Central Africa, Ibadan, Nigeria. Genetic Resources Multiplication and Utilization, 179 - 192, 2004.

LASSOUDIERE A., « Le bananier plantain en Côte d'Ivoire », Fruits. RESUME, 28(6), 453-462, 1973. 
LASSOUDIERE A., Enquête diagnostic sur la culture bananière, Préfecture de Kibungo, IRFA-CIRAD, ISAR, p 154, 1989.

LÓPEZ-NICOLÁS C., MEROÑO-CERDÁN Á. L., « Strategic knowledge management, innovation and performance », International journal of information management, 31(6), 502-509, 2011.

LUNDVALL B. A., National innovation system: towards a theory of innovation and interactive learning, Pinter, London. 1992.

MALERBA F., «Sectoral systems of innovation: a framework for linking innovation to the knowledge base, structure and dynamics of sectors », Economics of innovation and New Technology, 14(1-2), 63-82, 2005.

NEDERLOF S., WONGTSCHOWSKI M., VAN DER LEE F. (EDS), Putting heads together: Agricultural innovation platforms in practice, Bulletin 396. Amsterdam, the Netherlands: KIT Publishers Development, Policy \& Practice. 2011.

OSSENI, B., «Les systèmes de cultures comportant le bananier plantain en Côte d'Ivoire », In Banana Food Security Proceeding of INIBAP, International symposium. Douala (Cameroun), 10-14 November 1998, 689-693, 1998.

PERRIN, A., «Diagnostic de la filière Banane Plantain en Côte d'Ivoire », ONG RONGEAD_ International Trade \& Sustainable Development, Projet de Promotion et commercialisation de la Banane Plantain et du Manioc en Côte d'Ivoire financé par le Comité Français pour la Solidarité Internationale (CFSI), 66 p, 2015. http://www.rongead.org/IMG/pdf/diagnostic_de_la_filiere_banane_plantain_en_cote_d_ivoire_15_12_2015_final.pdf

PNIA, "PROGRAMME NATIONAL D'INVESTISSEMENT AGRICOLE", Document de plaidoyer 2010-2015, agriculture.gouv.ci. Rapport d'Expertise AISA, 118 p, Ministère en charge de l'Agriculture de Côte d'ivoire (MINAGRI), 2010. file:///C:/Users/Utilisateur/Downloads/PNIA\%20VERSION\%20FINALE.pdf

PNSAN, "PROGRAMME NATIONAL DE SECURITE ALIMENTAIRE ET DE NUTRITION", Rapport de Planification 2014-2020, Ministère en charge de l'Agriculture de Côte d'Ivoire (MINAGRI), Ministère en charge de la Santé et de la lutte contre le SIDA de Côte d'Ivoire et FAO_Organisation des Nations Unies pour l'alimentation et l'agriculture, 125 p, 2011.

RÖLING N., «Pathways for impact: scientists' different perspectives on agricultural innovation », International journal of agricultural sustainability, 7(2), 83-94. 2009. http://dx.doi.org/10.3763/ijas.2009.0043

SCHUT M., KLERKX L., SARTAS M., LAMERS D., MC CAMPBELL M., OGBONNA I., ... , LEEUWIS C., "Innovation platforms: experiences with their institutional embedding in agricultural research for development», Experimental Agriculture, 1-25, 2015.

SNDCV, "STRATEGIE NATIONALE DE DEVELOPPEMENT DES CULTURES VIVRIERES AUTRE QUE LE RIZ”, Rapport final de l'étude pour l'élaboration, Direction Générale de la Production et de la Sécurité alimentaire du Ministère en charge de l'Agriculture de Côte d'Ivoire (MINAGRI) et Union Européenne, Délégation Côte d'Ivoire. 83 p, 2014.

TEMPLE L., LANÇON F., PALPACUER F., PACHE, G., «Actualisation du concept de filière dans l'agriculture et l'agroalimentaire », Economies et sociétés, (33), 1785-1797, 2011.

TEMPLE L., KWA M., TETANG J., BIKOI A., «Organizational determinant of technological innovation in food agriculture and impacts on sustainable development », Agronomy for sustainable development, 31(4), 745-755, 2011.

TOUZARD J. M., TEMPLE L., FAURE, G., TRIOMPHE B., « Innovation systems and knowledge communities in the agriculture and agrifood sector: a literature review », Journal of Innovation Economics \& Management, 2, 117-142. 2015.

TRAORÉ S., KOBENAN K., KOUASSI S., GNONHOURI, G. P., « Plantain cultivation systems and pests management by smallholder producers in Côte d'Ivoire », Journal of Applied Biosciences, 19, 1094-1101, 2009.

WAAPP INFO5 "Bulletin d'informations du PPAAO / WAAPP Côte d'Ivoire - Numéro 5". « Journées agricoles en milieu paysan: Évaluation de 2 nouvelles variétés de banane plantain ». 2013. http://www.waappppaao.org/sites/default/files/bulletin_ci-5_1.pdf 